\title{
Inversionless light amplification and optical switching controlled by state-dependent alignment of molecules
}

\author{
A. K. Popow \\ Department of Physics \& Astronomy and Department of Chemistry, \\ University of Wisconsin-Stevens Point, Stevens Point, WI 54481, USA and \\ Institute of Physics of the Russian Academy of Sciences, 660036 Krasnoyarsk, Russia \\ V. V. Slabkd \\ Krasnoyarsk State Technical University, 660074 Krasnoyarsk, Russia and \\ Institute of Physics of the Russian Academy of Sciences, 660036 Krasnoyarsk, Russia
}

(Dated: October 6, 2003)

\begin{abstract}
We propose a method to achieve amplification without population inversion by anisotropic molecules whose orientation by an external electric field is state-dependent. It is based on decoupling of the lower-state molecules from the resonant light while the excited ones remain emitting. The suitable class of molecules is discussed, the equation for the gain factor is derived, and the magnitude of the inversionless amplification is estimated for the typical experimental conditions. Such switching of the sample from absorbing to amplifying via transparent state is shown to be possible both with the aid of dc and ac control electric fields.

PACS numbers: 42.55.-f, 33.55.-b, 33.80-b, 42.70.Hj
\end{abstract}

Amplification of light is determined by the difference between the net absorbtion and stimulated emission of radiation. Usually, this requires larger population of the upper quantum state then the lower one that are coupled by the induced transitions. However, the amount of absorbed and emitted photons depend not only on the populations of the resonant energy levels but also on the probabilities of the induced transitions and on distribution of the populations over the energy-degenerated states. Therefore, the inversion of population is, generally speaking, only a special case, in which the amount of the emitted light prevails over the absorbed one. Based on this fact, a variety of realizations of amplification without population inversion (AWI) was proposed in the early years of quantum electronics. Such possibility based on a possible difference in velocity distribution in upper and lower states was discussed in [1]. Nonreciprocity of probabilities of induced emission and absorbtion caused by nonlinear interference affect in the field of the auxiliary radiation was investigated for two-level atoms in [2] and for three-level systems in [3]. Corresponding AWI at transitions of neon was predicted and analyzed in details in [4] and proved in the experiments [5]. The asymmetry in the lineshape of net emission and absorption at the transitions to the autoionizing states and related possibility of AWI was considered in [6]. A possible AWI in dichroic molecules was pointed out in [7]. The feasibility of AWI of short pulses in three-level system was considered in 8]. A review of later publications on AWI is given in 9]. An asymmetry in absorption and emission lineshape of two-level system caused by its interaction with a thermostat is discussed in recent publication [10]. In this paper we propose and discuss a method of achieving lasing without population in- version by the anisotropic molecules. It bases on selective alignment and consequent decoupling the lower-state molecules from the polarized resonant radiation, while the upper-state molecules remain amplifying this radiation through its stimulated emission.

In electrodipole approximation, the probability of induced transitions between levels $m$ and $g, W_{m g}$, depends on the factor $d_{m g} \cos \theta$, which is the projection of the electrodipole transition matrix element on a direction of a vector of a resonant oscillating electric field that causes such transition,

$$
W_{m g}=B|E|^{2} F(\omega)
$$

Here, $B=8 \pi\left|d_{m g} \cos \theta\right|^{2} / \hbar^{2}$ is the Einstein coefficient, $F(\omega)$ is the transition form-factor, $\omega$ is frequency of radiation. For molecules, the direction of $\mathbf{d}_{m g}$ is stipulated by orientation of a molecule in space and by its symmetry. Under influence of an external dc electric field, a molecule turns towards the direction that corresponds to the minimum of potential energy of their interaction. Thus orientation of a molecule is determined by both the direction of an external field and by the symmetry of a molecule. The degree of alignment of a molecular medium depends on the alignment parameter, which is given by the ratio of the interaction energy with the external field $U$ to the energy of a thermal motion that renders disorientation. However, the energy of such interaction $U$, and consequently the degree of orientation, can be different for the molecules in lower and upper states. Therefore, in such case the probabilities of induced transitions corresponding to emission and absorption of polarized light, averaged over the molecules with different orientations, are not equal. This opens an opportunity of AWI for the polarized light through manipulating the difference 
in the orientation degree of the molecules at the different energy levels by the control field.

Consider amplification index $\alpha>0$ (absorption, if $\alpha<$ 0 ), which determines exponential change of intensity for the plain-polarized probe radiation along the medium $I=I_{0} \exp \{\alpha z\}$. In the presence of the control field $\mathbf{E}_{0}$, it is given by the equation

$$
\alpha=\sigma_{0} \int\left[n_{m} f_{m}\left(\Theta, \mathbf{E}_{0}\right)-n_{g} f_{g}\left(\Theta, \mathbf{E}_{0}\right)\right] \cos ^{2} \theta d \Theta .
$$

Here, $n_{m}$ and $n_{g}$ are populations of upper and lower levels created by any common way, $d \Theta=\sin \theta d \theta d \phi$ is element of a solid angle, and $\sigma_{0}=8\left|\pi d_{m g}\right|^{2} \omega F(\omega) / c \hbar$ is absorption/emission crosssection for the molecules, whose transition electrodipole moment is aligned along the polarization of the probe field $\mathbf{E}$.

The functions $f_{m}\left(\Theta, \mathbf{E}_{0}\right)$ and $f_{g}\left(\Theta, \mathbf{E}_{0}\right)$ describe the distribution of the molecules over orientations at upper $m$ and lower $g$ energy levels. They depend on the $U_{m, g}\left(\Theta, \mathbf{E}_{0}\right)$, the energy of their interactions with the control field $\mathbf{E}_{0}$ in the corresponding states. If the energy level lifetime excesses significantly the time required to set the orientation balance, they are given by the Boltzmann distribution

$$
f_{j}\left(\Theta, \mathbf{E}_{0}\right)=A_{j} \exp \left\{-U_{j}\left(\Theta, \mathbf{E}_{0}\right) / k T\right\} .
$$

Here, $A_{j}^{-1}=\int \exp \left\{-U_{j}\left(\Theta, \mathbf{E}_{0}\right) / k T\right\} d \Theta$ is a scale factor, $\mathrm{T}$ - temperature, $\mathrm{k}$ - Boltzmann constant, and $j=\{g, m\}$. Potential energy of molecules coupled with the electric field $\mathbf{E}_{0}$ can be written as follows [1]

$$
U_{j}\left(\Theta, \mathbf{E}_{0}\right)=\mu_{i}^{j} E_{0 i}-\beta_{i k}^{j} E_{0 i} E_{0 i k} .
$$

Here, $\mu_{i}^{j}$ is i-component of a vector of the permanent dipole moment and $\beta_{i k}^{j}$ is the component of the tensor of electrical polarizability, both for the molecules at energy level $j$. The first term in Eq. (4) represents the energy of molecules without center of symmetry, which possess a permanent dipole moment. The second term depicts the interaction energy with the dipole induced by the field $\mathbf{E}_{0}$. This term describes the alignment, which can be also caused by the strong optical field with the amplitude $\mathbf{E}_{0}$ and frequency $\omega_{0}[12]$.

Let's consider the example of axial-symmetric molecules and assume the directions of both permanent $\mu$ and of the electrodipole transition moment aligned along the symmetry axis of the molecule, which makes an angle $\theta_{0}$ with the control field $\mathbf{E}_{0}$. Then the interaction energy (4) and distribution functions (3) become dependent only on the angle $\theta_{0}$

$$
f_{j}\left(\theta_{0}, \mathbf{E}_{0}\right)=A_{j} \exp \left\{p_{j} \cos \theta_{0} \pm q_{j} \cos ^{2} \theta_{0}\right\} .
$$

Following 11], we introduce parameters of permanent dipole orientation $p_{j}$ and of the alignment determined by the polarizability ellipsoid $q_{j}$ as

$$
p_{j}=\mu^{j} E_{0} / k T, \quad q_{j}=\left(b_{33}^{j}-b_{11}^{j}\right) E_{0}^{2} / 2 k T .
$$

Here, $b_{33}^{j}$ and $b_{11}^{j}$ are principal values of the polarizability tensor along the symmetry axis and across it, accordingly, for the molecule at level $j$. For the control ac field $\mathbf{E}_{0}$ at $\omega_{0}$ the values $b_{33}$ and $b_{11}$ are given by equation 11

$$
b_{j}=\left(e^{2} / m\right) \sum_{l} d_{l j} /\left(\omega_{l j}^{2}-\omega_{0}^{2}\right)
$$

where $m$ and $e$ are mass and charge of electron, $\omega_{l j}$ is frequency and $d_{l j}$ is corresponding projection of the electrodipole moment of transition between levels $j$ and $l$.

We shall illustrate the effect of inversionless amplification for two cases $\mathbf{E} \uparrow \uparrow \mathbf{E}_{0}$ and $\mathbf{E} \perp \mathbf{E}_{0}$ depicted in Figs. 11 and 3 . where the molecule symmetry axis $\mathbf{C}, \mu_{m, g}$, and $\mathbf{d}_{\mathbf{m g}}$ coincide. If the permanent dipole moment in the upper energy level is larger, than in the lower one $\left(\mu_{m}>\mu_{g}\right)$, then AWI occurs for $\mathbf{E}$ polarized along $\mathbf{E}_{0}$. In this case $\theta=\theta_{0}$, and Eq. (2) takes the form

$$
\alpha=\sigma_{0}\left[n_{m}\left(1-\frac{2}{p_{m}}\right) L\left(p_{m}\right)-n_{g}\left(1-\frac{2}{p_{g}}\right) L\left(p_{g}\right)\right],
$$

where $L\left(p_{j}\right)$ is Langevin function [11, 13.

$$
\begin{aligned}
& L\left(p_{j}\right)=\operatorname{coth} p_{j}-\frac{1}{p_{j}}=\left(1-\frac{2}{p_{j}}\right)^{-1} \\
& \times \int_{0}^{2 \pi} d \varphi \int_{0}^{\pi} d \theta_{0} A_{j} \exp \left\{p_{j} \cos \theta_{0}\right\} \cos ^{2} \theta_{0} \sin \theta_{0} .
\end{aligned}
$$

Figure 1 shows the existence of the optimum value of

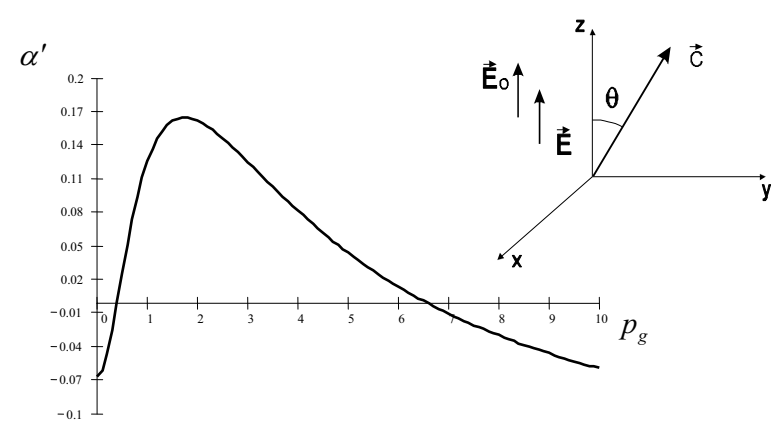

FIG. 1: Scaled amplification index $\alpha^{\prime}=\alpha / n_{g} \sigma_{0}$ vs alignment parameter $p_{g}=\mu_{g} E_{0} / k T$ at $\mu_{m} / \mu_{g}=4, n_{m} / n_{g}=0.8(\mathbf{E} \uparrow \uparrow$ $\mathbf{E}_{0}$ ). Orientations of the probe field $\mathbf{E}$, molecule symmetry axis $\mathbf{C}$ and of the control field $\mathbf{E}_{0}$ are depicted in the inset.

the alignment parameter $p_{g}$ (i.e., $\left.E_{0}\right)$ for the fixed values $\mu_{m} / \mu_{g}$ and $n_{m} / n_{g}$, while Fig. 2 shows that the same amplification index can be achieved for different sets of population and alignment parameters.

In the alternative case, where the dipole moment in the upper state is less than in the lower one $\left(\mu_{m}<\mu_{g}\right)$, the alignment degree in the lower state is larger than for the molecules in the upper state. Consequently, orthogonal orientation of polarizations of the probe $\mathbf{E}$ and control 


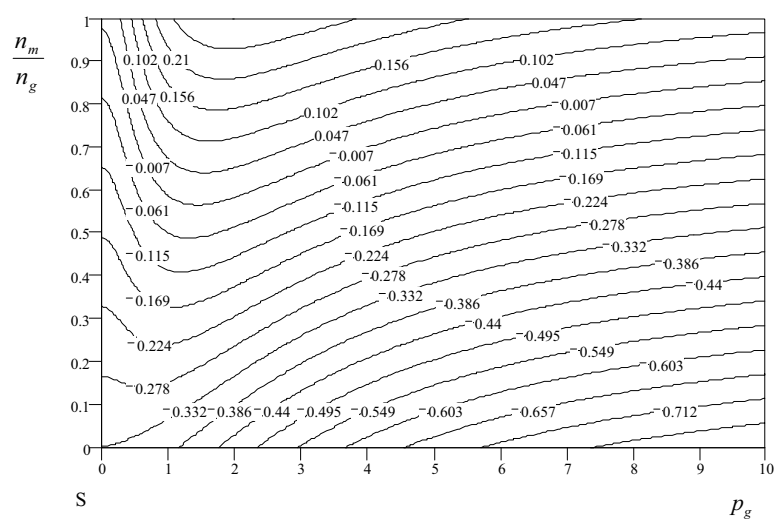

FIG. 2: Equal values of scaled amplification index $\alpha^{\prime}=$ $\alpha / n_{g} \sigma_{0}$ at $\mu_{m} / \mu_{g}=4\left(\mathbf{E} \uparrow \mathbf{E}_{0}\right)$.

$\mathbf{E}_{0}$ fields becomes advantageous for the suppression of absorption. Then the amplification index averaged over the molecule orientation is given by the equation

$$
\alpha=\sigma_{0}\left\{\left[n_{m} L\left(p_{m}\right) / p_{m}\right]-\left[n_{g} L\left(p_{g}\right) / p_{g}\right]\right\} .
$$

Note, that contrary to the previous case, AWI becomes

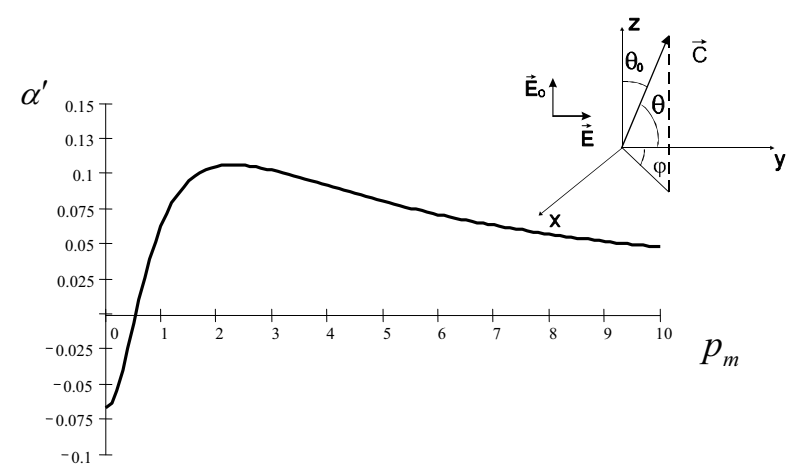

FIG. 3: Scaled amplification index $\alpha^{\prime}=\alpha / n_{g} \sigma_{0}$ vs alignment parameter $p_{m}=\mu_{m} E_{0} / k T$ at $\mu_{g} / \mu_{m}=4, n_{m} / n_{g}=0.8(\mathbf{E} \perp$ $\left.\mathbf{E}_{0}\right)$. Orientations of the probe field $\mathbf{E}$, molecule symmetry axis $\mathbf{C}$ and of the control field $\mathbf{E}_{0}$ are depicted in the inset.

possible at any strength of $E_{0}$ above the threshold value, which depends on the specific magnitude of the population ratio $n_{m} / n_{g}$ (Fig. 3). Corresponding changes occur in the sets of parameters attributed to one and the same

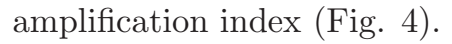

Similar conclusions are valid for AWI induced by an ac field $E_{0}$, which is stipulated by the difference of the induced dipole moments in the upper and lower states.

We shall discuss further the suitable types of molecules and important related features. As an example, the molecule 3-6-Diacetyl-amino-phtalimide can be considered because it does not possess permanent dipole moment in the excited state, i.e., $\mu_{m}=0$, while $\mu_{g} \approx 5.5 \mathrm{D}$

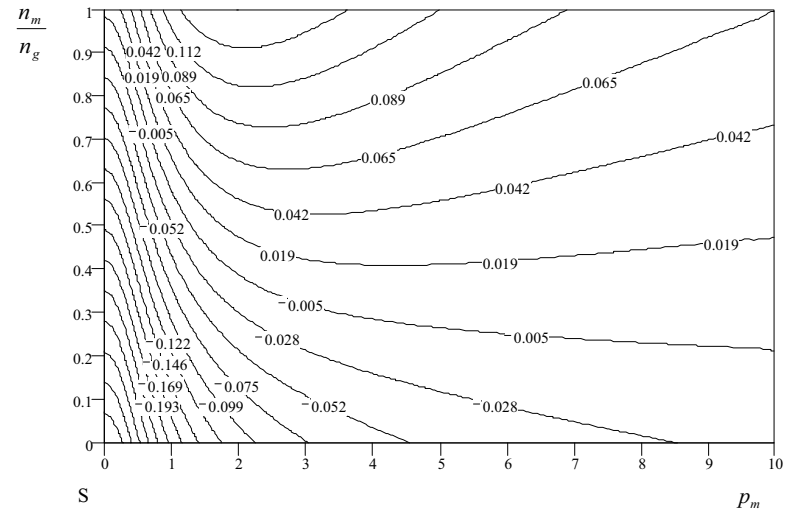

FIG. 4: Equal values of the scaled amplification index $\alpha^{\prime}=$ $\alpha / n_{g} \sigma_{0}$ at $\mu_{g} / \mu_{m}=4\left(\mathbf{E} \perp \mathbf{E}_{0}\right)$.

[14]. In this case, at $p_{g} \rightarrow \infty$, and $\mathbf{E} \perp \mathbf{E}_{0}$, amplification is determined only by the upper-state molecules and does not depend on population of the lower state:

$$
\alpha=\sigma_{0} n_{m} / 3 .
$$

This is because the lower-state molecules are aligned orthogonal to the the probe field and, therefore, are not coupled with this field. On the contrary, the excited molecules are decoupled from the control field $E_{0}$, their orientation remains isotropic in dipole approximation and, hence, the averaged projection of transition dipole moment $\mathbf{d}_{m g}$ on the probe field $\mathbf{E}$ is not equal to zero.

The important requirement for the realization of the proposed technique is that the lifetime in the excited state $\tau$ must exceed the time $\tau_{0}$ necessary to set the orientation equilibrium. This is because the molecules aligned at ground state must achieve a new distribution, corresponding to the alignment parameter at the upper state, after excitation to that state. The characteristic lifetime for the electrodipole transition is $\tau \simeq 10^{-8}-10^{-9} \mathrm{~s}$. The alignment time may vary in the wide range depending on the molecule size and on the viscosity of the solvent. For big organic molecules in the solvents the characteristic time is $\tau_{0} \simeq 10^{-10}-10^{-12} \mathrm{~s}$, for protein macromolecules $\tau_{0} \simeq 10^{-6}-10^{-8} \mathrm{~s}$, and $\tau_{0} \simeq 10^{-2}-10^{-4} \mathrm{~s}$ for big biomacromolecules. Thus, for the applications being discussed here the molecule mass must not significantly exceed $10^{3}$ atomic units and their length - some $10 \AA$. For such compounds the constant electric dipole moment is usually on the order of $1-10 \mathrm{D}[11,14,15]$. Therefore the alignment parameter achieved with the nearly breakdown dc field $E_{0}$ can hardly exceed the value about unit, even in the most electrically resistant solvents [16]. However, the breakdown threshold can be increased by the use of the pulsed dc fields $E_{0}$ and by the decrease of the temperature of the solvents, which is possible making use of cryogenic liquids or cooled gas. The alignment parameters above the unit seem to be achieved by ap- 
plying a dc field $E_{0}$ to relatively large molecules having dipole moment on the order of $10^{2}-10^{3} \mathrm{D}$. However, their reorientation time $\tau_{0}$ most likely is longer than the lifetime at the exited state. Thus, the effect of AWI, apparently, may become easier to achieve through orientation of molecules by ac optical field.

In the case of optical orientation, the polarizability anisotropy $b_{33}-b_{11}$ is about $10^{-23} \mathrm{~cm}^{3}$ even for the resonance detuning on the order of $\left|\omega_{i j}-\omega_{0}\right| \simeq \omega_{0}$. At normal temperature $\mathrm{T}=300 \mathrm{~K}$, optical alignment parameter $q$ defined by Eq. (6) can reach the magnitude about unit under focused laser radiation of the power about $10^{6}$ W. The increase of intensity of the control field up the breakdown threshold allows one to achieve the complete optical orientation of molecules, with the size and weight not exceeding the magnitudes discussed above. Besides that, the magnitude of $q$ can be considerably increased by setting the frequency $\omega_{0}$ in the vicinity of of the resonant frequencies of the adjacent optical transitions.

In summary, we propose to achieve amplification and lasing without population inversion by anisotropic molecules through decoupling polarized light from the absorbing molecules at lower energy level while the upperstate molecules stay emitting. Such switching becomes feasible with the aid of control electric field that aligns anisotropic molecules in upper and lower energy levels in a different way. The example of suitable molecules similar to dye molecules is given and the favorable conditions are discussed and illustrated. The achievable magnitudes of amplification without population inversion are estimated based on the derived equations.

We thank V. V. Laschinsky for help with computing and drawing the graphs.

\footnotetext{
* Corresponding author: Alexander Popov; Electronic address: apopov@uwsp.edu URL: http://www.kirensky.ru/popov

$\dagger$ Electronic address: slabko@iph.krasn.ru
}

[1] S. G. Rautian and P. L. Rubin, Optika i Spektroscopiya, 18, 326 (1965) (in Russ); M. L. Sosinskii and E. N. Morozov, ibid, 510 (1965).

[2] S. G. Rautian and I. I. Sobelman, JETP 14, 328 (1962).
[3] G. E. Notkin, S. G. Rautian, and A. A. Feoktistov, JETP 25, 1112 (1967); T. Ya. Popova, A. K. Popov, S. G. Rautian and A. A. Feoktistov, Zh. Eksp. Teor. Fiz. 57, 444 (1969), [Sov. Phys. JETP 30, 243 (1970)] (http://xxx.lanl.gov/abs/quant-ph/0005081);

T. Ya. Popova, A. K. Popov, S. G. Rautian, and R. I. Sokolovskii, Zh. Eksp. Teor. $\left.\begin{array}{llllllll}\text { Fiz. } & 57 & 850 & (1969)\end{array}\right]$ (http://xxx.lanl.gov/abs/quant-ph/0005094).

[4] T. Ya. Popova and A. K. Popov, Zhurn. Prikl. Spektrosk. 12, 989 (1970) [Transl. in Engl.: J. Appl. Spectr. 12, 734 (1970)] (http://xxx.lanl.gov/abs/quant-ph/0005047); T. Ya. Popova and A. K. Popov, Izv. Vysh. Uchebn. Zaved., Fizika No 11, (1970) 38 [Transl. in Engl.: Soviet Phys. J. 13, 1435 (1970)] (http://xxx.lanl.gov/abs/quant-ph/0005049); A.K. Popov, Vvedenie v Nelineinuyu Spektroskopiyu (Novosibirsk: Nauka, 1983)(in Russ).

[5] I. M. Beterov, Cand. Sci. Dissertation,Institute of Semiconductor Physics, Novosibirsk, Dec. 1970.

[6] V. G. Arkhipkin and Yu. I. Heller, Phys. Lett. A98, 12 (1983).

[7] A. K. Popov and V. V. Slabko, On possibility of inversionless amplification of light by dichroic molecules, Preprint 410F, Institute of Physics SD USSR AS, Krasnoyarsk, 1986.

[8] O. Kocharovskaya and Ya. I. Khanin, Pis'ma v Zh. Eksp. Teor. Fiz. 48, 581 (1988) [JETP Lett. 48, 630 (1988)].

[9] J. Mompart and R. Corbalan, J. Opt. B: Quantum Semiclass. Opt. 2, R7 (2000).

[10] A. M. Shalagin, JETP Letters 75, 253 (2002).

[11] S. Kielich, Molecular nonlinear optics, Moscow, Nauka, 1981, (Russ); J. R. Lalanne, Andre Ducasse, S. Kielich, Laser-Molecule Interaction: Laser Physics and Molecular Nonlinear Optics, Willey, 1996.

[12] H. Stapelfeldt, Rev. Mod. Phys. 75, 543 (2003).

[13] E. Jahnke, F. Emde and F. Loesch, Special functions, Moscow, Nauka, 1977, (in Russ); E. Jahnke and F. Emde, Tables of Functions with Formulae and Curves, 4th ed. New York: Dover, 1945.

[14] A. N. Terenin, Photonics of dye molecules and allied organic compounds, Leningrad, Nauka, 1967, (in Russ).

[15] N. G. Bakhshiev, Spectroscopy of intermolecular interactions, Leningrad, Nauka, 1972, (in Russ); A. N. Vereschagin, Characteristics of anizotropy of polarizability of molecules, Moscow, Nauka, 1982, (Russ).

[16] Tables of physical values, ed. by I. K. Kikoin, Moscow, atomizdat, 1976, (in Russ). 